\title{
A neutron beam facility at SPIRAL-2
}

X. Ledoux ${ }^{1}$, M. Aïche ${ }^{2}$, G. Ban ${ }^{3}$, G. Barreau ${ }^{2}$, P. Baumann ${ }^{4}$, P. Bem ${ }^{5}$, V. Blideanu ${ }^{6}$, J. Blomgren ${ }^{7}$, S. Czajkowski $^{2}$, P. Dessagne ${ }^{4}$, E. Dupont ${ }^{6}$, D. Doré ${ }^{6}$, T. Ethvignot ${ }^{1}$, U. Fischer ${ }^{8}$, F. Gunsing ${ }^{6}$, B. Jacquot ${ }^{9}$, B. Jurado ${ }^{2}$, M. Kerveno $^{4}$, F.R. Lecolley ${ }^{3}$, J.L. Lecouey ${ }^{4}$, F. Negoita ${ }^{10}$, S. Oberstedt ${ }^{11}$, M. Petrascu ${ }^{10}$, A.J.M. Plompen ${ }^{11}$, F. Rejmund ${ }^{9}$, D. Ridikas ${ }^{6}$, G. Rudolf ${ }^{4}$, O. Shcherbakov ${ }^{12}$, S.P. Simakov ${ }^{8}$, and J. Taïeb ${ }^{1}$

1 CEA/DIF, DPTA/SPN, BP. 12, 91980 Bruyères-le-Châtel Cedex, France

2 Centre d'Études Nucléaires de Bordeaux-Gradignan, 33175 Gradignan, France

3 LPC, ISMRa et Université de Caen, CNRS/IN2P3, France

${ }^{4}$ Institut Pluridisciplinaire Hubert Curien, Strasbourg, France

5 Nuclear Physics Institute, 25068 Řež, Czech Republic

6 CEA Saclay, DSM/DAPNIA, 91191 Gif-sur-Yvette, France

7 Department of Neutron Research, Uppsala University, Uppsala, Sweden

${ }^{8}$ Forschungszentrum Karlsruhe, Institute for Reactor Safety, Karlsruhe, Germany

9 GANIL, CEA/DSM-CNRS/IN2P3, Caen, France

10 NIPNE, Bucharest, Romania

11 European Commission, JRC/IRMM, Geel, Belgium

12 PNPI, Gatchina, Leningrad district, Russia

\begin{abstract}
The future SPIRAL-2 facility is mainly composed of a high-power superconducting driver LINAC, delivering a high-intensity deuteron, proton and heavy ions beams. The first two beams are particularly well suited to the construction of a neutron beam and irradiation facility called Neutrons for Science (NFS). Thick C and Be target-converters with incident deuteron beam will produce an intense white neutron spectrum, while thin ${ }^{7} \mathrm{Li}$ target and incident proton beam allows generating quasi-monoenergetic neutrons. The $1-40 \mathrm{MeV}$ neutron energy range will be covered and characterized by very intense fluxes available. The primary ion beam characteristics (energy, time resolution, intensity, etc.) are adequate to create a neutron time-of-flight facility. Irradiation stations for neutron, proton and deuteron induced reactions could also be built in order to perform cross-sections measurements by activation techniques. In this paper we will discuss the potential of this new installation to investigate numerous topics, both in fundamental and applied physics. In particular, cross section measurements could be performed for different purposes like nuclear data evaluation, fission and fusion technology, Accelerator Driven Systems, nuclear medicine, astrophysics, etc.
\end{abstract}

\section{Introduction}

The future SPIRAL-2 facility, currently under construction at GANIL, Caen (France) will produce radioactive ion beam (RIB) in the mass range from $\mathrm{A}=60$ to $\mathrm{A}=140$. These nuclei will be produced by the fission of ${ }^{238} \mathrm{U}$ induced by fast neutrons, which are generated from deuterons interacting with a carbon converter [1]. The high-power superconducting driver LINAG (LINear Accelerator of Ganil) will deliver a high-intensity deuteron as well as proton and heavy ions beams. The delivered beams will also be used for other purposes than RIB production [2]. We can mention the atomic physic facility or the $S^{3}$ experiment where high intensity heavy ions beams will be used for super-heavy studies. Finally, LINAG's characteristics are also particularly well suited to the construction of a neutron beam and irradiation facility, called Neutrons for Science (NFS). This facility will be a very powerful tool for physics with neutron beams from fundamental research to applications like the transmutation of nuclear waste, design of future fission and fusion reactors, nuclear medicine or the test and development of new detectors. We will describe in this paper the technical characteristics of the facility and give some examples of the physics case which could be achieved.

\section{Description of NFS}

\subsection{The neutron hall}

The NFS project will be composed of two parts: a pulsed neutron beam for in-flight measurements and an irradiation station for activation measurements. The facility will be composed of the primary proton/deuteron beam extension in a dedicated cave with a target converter (neutron production target). Behind the converter a thick concrete wall with a collimated channel defines the neutron beam (see figure 1). The size of the neutron hall downstream of the collimator will be around

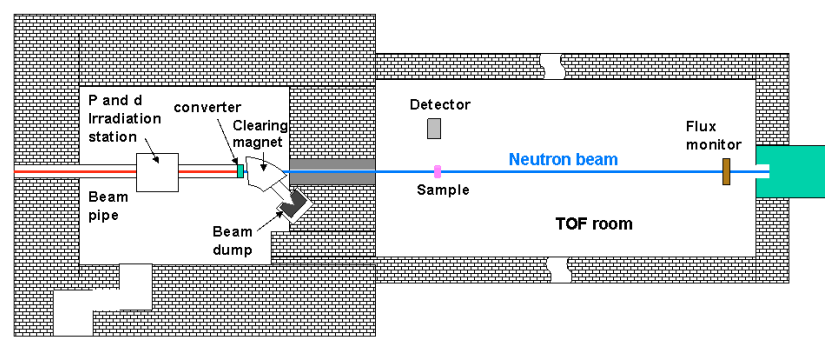

Fig. 1. Schematic view of the NFS facility. 
$\mathrm{L} \sim 25 \mathrm{~m} \times 1 \sim 8 \mathrm{~m}$. This size would allow using large experimental set-ups and performing measurements at desired distances from 5 up to $30 \mathrm{~m}$. This flexibility is very interesting in terms of flux and energy measurement resolution (see sect. 1.3). The clearing magnet placed between the converter and the collimator allows the deviation of an outgoing beam (in case of thin converter) to the beam dump and clean up of the neutron beam from the secondary charged particles created in the converter.

\subsection{Neutron production}

The LINAG is designed to accelerate deuterons up to $40 \mathrm{MeV}$ and protons up to $33 \mathrm{MeV}$. The accelerator frequency is $88 \mathrm{MHz}$, the burst duration is $200 \mathrm{ps}$ and the nominal intensity is $5 \mathrm{~mA}$. Two types of production reactions are conceivable to produce neutrons.

The first is the deuteron break-up reaction on $1 \mathrm{~cm}$ thick converter. A continuous spectrum is generated with an average energy of around $14 \mathrm{MeV}$ at 0 degrees and the impinging deuteron is stopped in the target. We can see on figure 2 that the use of beryllium instead carbon allows to gain a factor of 2 in the neutrons yield. The second production mode is obtained by the ${ }^{7} \mathrm{Li}(\mathrm{p}, \mathrm{n}){ }^{7} \mathrm{Be}$ reaction on a thin target $(\sim 1 \mathrm{~mm})$. Quasi-monoenergetic neutrons are produced at 0 degree with energy $E_{n} \approx E_{p}-2 \mathrm{MeV}$ (see fig. 3). The energy resolution and the low energy tail depend mainly on the lithium target thickness. A pulsed beam is required to measure the neutron energy in case of continuous spectrum and to separate quasimono-energetic neutrons from the low energy tail.

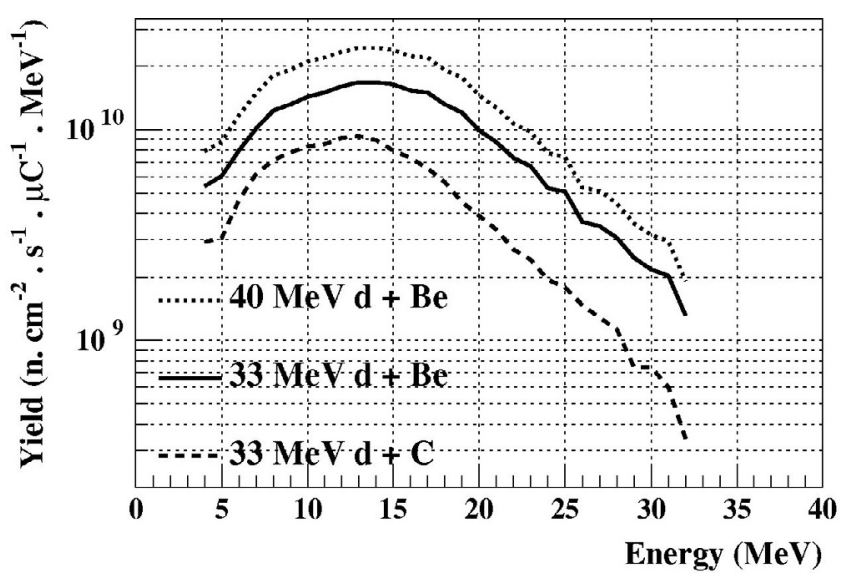

Fig. 2. Neutron spectra produced at 0 degree by deuteron break-up reactions on $1 \mathrm{~cm}$ carbon and beryllium converters [4].

\subsection{Neutron beam characteristics}

Neutron energy resolution and minimum available energy are two very important characteristics of a neutron beam facility. They are directly related to the flight path, the burst duration and the repetition frequency. Due to the beam transport between the LINAG exit and the converter, the initial burst of 200 ps will be somewhat degraded at the converter

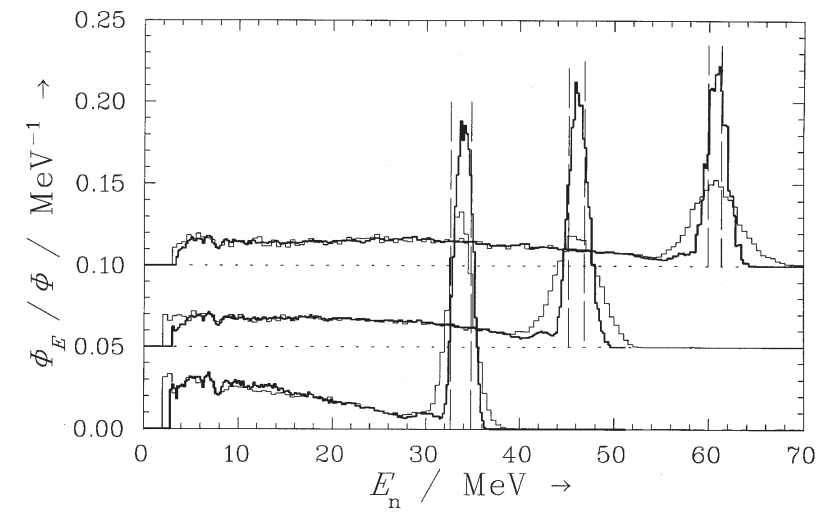

Fig. 3. Neutron spectra produced at 0 degree by ${ }^{7} \mathrm{Li}(\mathrm{p}, \mathrm{n}){ }^{7} \mathrm{Be}$ reaction at 36,48 and $63 \mathrm{MeV}$ [5].

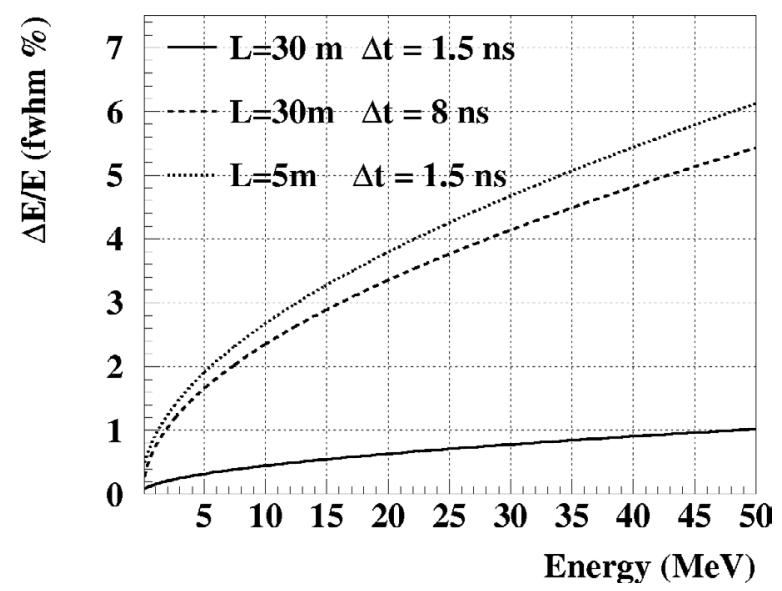

Fig. 4. Energy resolution as a function of the neutron energy.

point. However, a burst duration better than $1 \mathrm{~ns}$ should be guaranteed by adding a beam buncher if necessary. Taking into account a $30 \mathrm{~m}$ flight path and variable time resolution of different detectors we can calculate the expected resolution on the energy measurement (see eq. 1 and fig. 4).

$$
\frac{\Delta E}{E}=\gamma(\gamma+1) \sqrt{\left(\frac{\Delta t}{t}\right)^{2}+\left(\frac{\Delta L}{L}\right)^{2}} .
$$

It can be observed that with fast detectors the energy resolution at $40 \mathrm{MeV}$ is better than $1 \%$ and even for slow detectors like HPGe $(\Delta \mathrm{t} \approx 8 \mathrm{~ns})$ the energy resolution remains below than $5 \%$.

The overlap of neutron time-of-flight $t$ from the one of the previous burst with TOF $\mathrm{t}+\mathrm{T}$ ( $\mathrm{T}$ is the beam period) limits the available energy range. The period $\mathrm{T}$ and the flight path L define the lowest usable energy (threshold energy). For our purpose a unique burst selector is required to divide the initial LINAG frequency $\left(\mathrm{F}_{0}=88 \mathrm{MHz}\right)$ by a factor $\mathrm{N}$. The intensity on the converter is then limited to $\mathrm{I}=5 \mathrm{~mA} / \mathrm{N}$. The table 1 gives some examples of beam frequency and maximum intensity for several path length and threshold.

By taking into account the neutron yield production, the beam division and the flight path, the neutron flux can be evaluated and compared to other major time-of-flight facilities 
Table 1. Time structure parameters and maximum energy for different path lengths and energy threshold.

\begin{tabular}{lcccc}
\hline Path length & E threshold & Frequency & N & Imax \\
\hline $5 \mathrm{~m}$ & $1 \mathrm{keV}$ & $88 \mathrm{kHz}$ & 1000 & $5 \mu \mathrm{A}$ \\
$5 \mathrm{~m}$ & $100 \mathrm{keV}$ & $875 \mathrm{kHz}$ & 100 & $5 \mu \mathrm{A}$ \\
$30 \mathrm{~m}$ & $100 \mathrm{keV}$ & $146 \mathrm{kHz}$ & 603 & $8.3 \mu \mathrm{A}$ \\
$30 \mathrm{~m}$ & $1 \mathrm{MeV}$ & $461 \mathrm{kHz}$ & 191 & $26.2 \mu \mathrm{A}$ \\
\hline
\end{tabular}

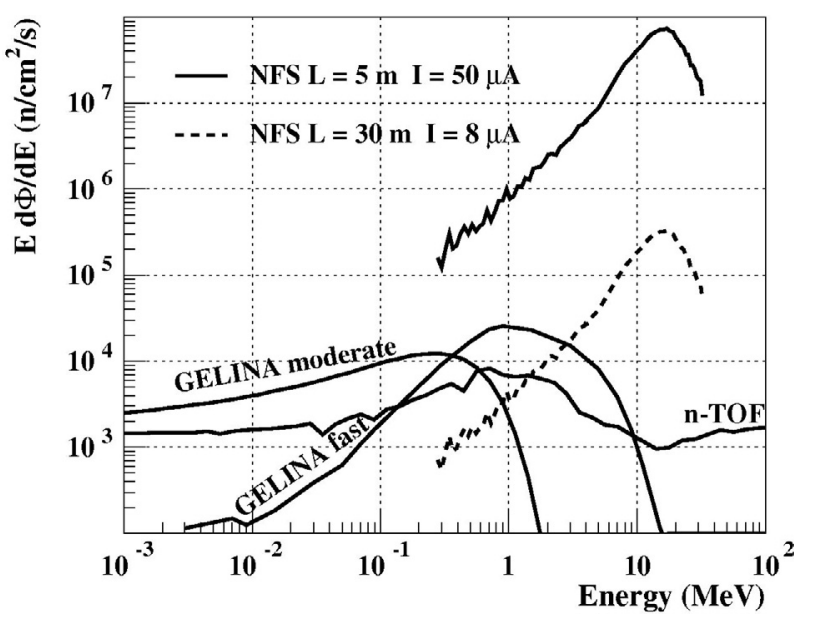

Fig. 5. Neutron flux at NFS for short $(5 \mathrm{~m})$ and long $(30 \mathrm{~m})$ compared to 2 other $n$-tof facilities in Europe.

in Europe namely n_TOF at CERN and Gelina at Geel. A flight path between 5 and $30 \mathrm{~m}$ is available at NFS allowing high intensity flux $(5 \mathrm{~m})$ and high resolution measurement $(30 \mathrm{~m})$. We can see on figure 5 that between 1 and $35 \mathrm{MeV}$ NFS is very competitive in terms of average flux in comparison with $\mathrm{n}_{-} \mathrm{TOF}$ and Gelina. It has to be stressed that it is mainly due to the high repetition rate, the flux by deuteron burst is clearly lower.

Moreover, NFS presents some advantages thanks to the neutron production mechanism itself. In spallation sources, the high energy neutrons (up to hundreds $\mathrm{MeV}$ ), can present challenges for collimation and background. Secondly the gammaflash, which is known to be very penalising especially because it induces a dead time, will be probably strongly reduced at NFS. Note that high energy gammas are produced by $\pi^{0}$ decay in spallation sources and by bremsstrahlung process in photoneutron sources based on electron accelerator.

\subsection{Irradiation station}

A station dedicated to measurements by activation technique for neutron and deuteron induced reactions is also envisaged. The irradiation sample should be placed as close as possible to the converter in order to maximize available neutron/deuteron flux. No time structure is required in this case and the maximum ion beam intensity will be limited to around $50 \mu \mathrm{A}$ in order to reduce the radioprotection constraints and make the converter design easier. For quasi-mono-energetic neutrons replacing the magnet by a beam stopper made of carbon seems

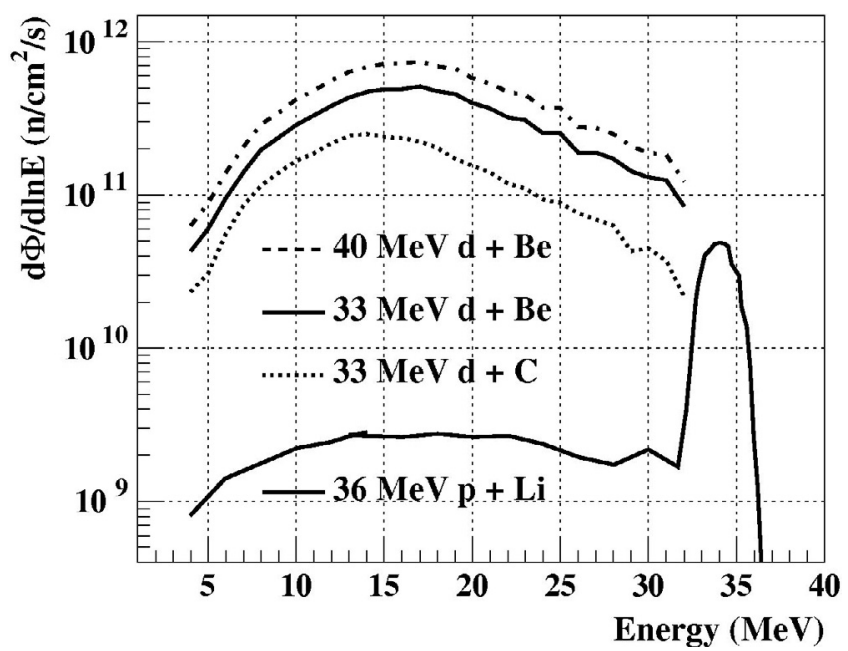

Fig. 6. Available neutron flux for activation experiments.

to be a more adequate solution since in this case the sample could be positioned closer to the Li target. In figure 6 the neutron flux available close to the converter $(5 \mathrm{~cm})$ for a beam intensity of $50 \mu \mathrm{A}$ is presented. Note that even at this reduced intensity the available neutron fluxes are higher than in the existing neutron facilities in Europe, where irradiations by high energy neutrons are performed.

The LINAG offers the possibility of delivering proton and deuteron beams with variable energy up to 33 and $40 \mathrm{MeV}$ respectively. Thus a charged particle irradiation station can be created without major difficulties, although it requires a specific set-up to place and remove the sample, and measure the beam current.

\section{Physics case}

The NFS facility could be used for cross-section measurements as well as for fundamental research [6,7]. The $1-40 \mathrm{MeV}$ energy range is particularly well suited for studies on the transmutation of nuclear waste in ADS or in the new generation fast reactors.

Required fission cross-sections are often unknown above $14 \mathrm{MeV}$ (or known with big uncertainties). A high neutron flux is absolutely needed because for the actinides of interest being radioactive only small samples can be used. The study of the fission process for fundamental research could also be achieved. Coincidence experiments could be performed, where the fragment mass and charge distribution could be measured. The variable neutron energy allows studying the process at variable excitation energies during the same experiment.

The $(n, X)$ reactions are also of first importance in numerous applications. We saw that measurements using germanium detector are possible with good energy resolution, so (n,n' $\gamma)$ and $(n, x n)$ can be studied. The impact of such reactions in large systems as nuclear reactors is very important on the energy distribution of the fast neutron spectrum. The NFS energy range allows one to access the opening of new reaction channels like $(n, 2 n),(n, 3 n),(n, 4 n) \ldots$ This is also the region 
where the pre-equilibrium process becomes important and measurements are needed to constrain strongly the existing models. The production of charged particles is of primary importance too because it leads to the generation of gases and defaults in the structural materials. If double differential measurements have been performed above $40 \mathrm{MeV}$, presently no data exist below this energy. It seems that the existing experimental apparatus could be used at NFS.

The fusion technology projects like IFMIF and ITER require activation cross-section measurements in fast neutron, proton and deuteron induced reactions $[8,9]$. These data are absolutely needed to determine the nuclear safety issues such as gas production, $\gamma$-dose rate, heat generation, waste transportation and storage. Such measurements could be achieved by activation techniques in the dedicated irradiation stations.

The neutrons produced in atmosphere by cosmic particles can produce single event upsets (SEUs) in semi-conductor devices on board of aircrafts or satellites. Accurate dosimetric measurements are an important tool for understanding the rate of (SEUs) in semi-conductor devices in neutron environments. The NFS irradiation station would allow performing such measurements in particular with quasi-mono-energetic neutrons.

\section{Conclusions}

The NFS characteristics in terms of flux or energy resolution make it a very attractive and powerful tool for neutron physics in the $1-40 \mathrm{MeV}$ range. The high intensity neutron beam will allow performing cross section measurements as well as fundamental physics experiments. This facility is fully complementary to the other existing facilities based on spallation neutron source or electron accelerators. The irradiation facility is particularly well situated for cross-section measurements in neutron, proton or deuteron induced reactions which are needed for the fusion technology. A letter of intent has been presented to the Scientific Advisory Committee of SPIRAL 2 [10] and received encouraging recommendations. The facility could be operational around 2011 .

\section{References}

1. Report of the SPIRAL 2 Detail Design Study, available on http://www;ganil.fr.

2. The scientific objective of the SPIRAL-2 project, available on http://www;ganil.fr.

3. D. Ridikas, X. Ledoux et al., Neutrons for Science (NfS) at SPIRAL-2, Internal report DAPNIA 05-30, CEA Saclay, France (2005).

4. Meulders et al., Phys. Med. Biol. 20(2), 235 (1975).

5. Schumacher et al., NIM A 421, 2843 (1999).

6. International Workshop on Neutrons for Science (NFS) at SPIRAL-2, GANIL, Caen, France, 13-14 December 2004.

7. A. Plompen, Nuclear Data Needs for Nuclear Energy (fission) and Possible Contributions of SPIRAL2, $15^{\text {th }}$ Colloque GANIL, Giens, France (2006).

8. U. Fischer, Nuclear data needs for fusion technology and possible contribution by SPIRAL2, $15^{\text {th }}$ Colloque GANIL, Giens, France (2006).

9. P. Bem et al., Activation cross section benchmarks at NPI cyclotron, Workshop on Activation Data - EAF 2007, 02-04 October 2006, Prague, Czech Republic.

10. Letters of intents for the SPIRAL 2 facility, available on http://www;ganil.fr. 